\title{
Plantas lenhosas da Floresta Ombrófila Mista do Parque Municipal das Araucárias - Guarapuava (PR)
}

\author{
Woody plants of the Mixed Ombrophylous Forest the Araucaria Municipal \\ Park - Guarapuava (PR)
}

\author{
Juliano Cordeiro ${ }^{1(*)}$ \\ Carlos Vellozo Roderjan ${ }^{2}$ \\ William Antonio Rodrigues ${ }^{3}$
}

\section{Resumo}

Foi realizado o levantamento florístico das espécies lenhosas da uma área de 41 ha de Floresta Ombrófila Mista (FOM) do Parque Municipal das Araucárias, localizado

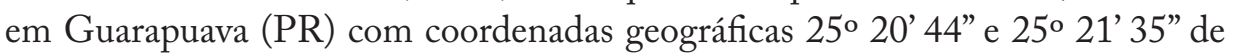

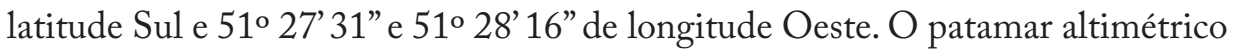
do Parque fica em torno dos 1070 m.s.n.m., o predomínio é relevo suave ondulado e solo do tipo Latossolo Bruno Ácrico Húmico. O levantamento foi realizado de mar/04 a out/08 com média de uma visita a cada vinte dias. Foram identificadas 107 espécies, pertencentes a 77 gêneros e 41 famílias botânicas, distribuídos nas formas biológicas de microfanerófitos ( $n=44)$, mesofanerófitos $(n=39)$, nanofanerófitos $(n=15)$, lianas $(n=8)$ e megafanerófito $(n=1)$. As famílias que apresentaram maior diversidade foram: Solanaceae com 12 espécies, Myrtaceae com nove, Fabaceae com oito, Bignoniaceae com seis, Asteraceae com cinco. Estas famílias agruparam $37,4 \%$ das espécies, enquanto que as outras 38 famílias responderam por $62,6 \%$ da diversidade. Aproximadamente $60,5 \%$ das famílias são representadas por apenas uma espécie cada. Considerando o critério a frequência Allophylus edulis (A. St.Hil., A. Juss \& Cambess.) Hieron. ex Niederl., Casearia decandra Jacq., Cedrela fissilis Vell., Cinnamodendron dinisii Schwacke, Jacaranda puberula Cham., Matayba elaeagnoides Radlk., Ocotea porosa (Nees \& Mart.) Barroso, Ocotea puberula (Rich.) Nees, Ocotea pulchella (Nees) Mez, Schinus terebinthifolius Raddi, Vernonanthura discolor (Spreng.) H. Rob. e Zanthoxylum rboifolium Lam. podem ser consideradas espécies companheiras da Araucaria angustifolia (Bertol.) Kuntze na florística da

I Dr.; Biólogo; Professor da Universidade Federal do Paraná, UFPR, Campus de Palotina; Endereço: Rua Pioneiro, 2153,Bloco Didático III,Jardim Dallas, CEP:85950-000,Palotina,Paraná,Brasil;E-mail:julianocordeiro@ufpr.br

2 Dr.; Engenheiro Florestal; Professor do Departamento de Ciências Florestais da Universidade Federal do Paraná, UFPR; Endereço: Av. Prefeito Lothário Meissner, 3400, CEP: 80.210-170, Curitiba, Paraná, Brasil; E-mail: roderjan@ufpr.br

3 Dr.; História Natural; Professor Sênior do Departamento de Botânica da Universidade Federal do Paraná, UFPR; Caixa Postal 1903I, CEP: 8I.53I-970, Curitiba, Paraná, Brasil; Bolsista de Produtividade em Pesquisa do CNPq; E-mail: william@ufpr.br

Recebido para publicação em I2/04/20 I I e aceito em I6/08/20I I

\begin{tabular}{llllll}
\hline Ambiência Guarapuava (PR) & v.7 n.3 & p. 44I - 460 & Set./Dez. 20II & ISSN I808 - 025I
\end{tabular}

DOI: $10.5777 /$ ambiencia. 2011.03 .03 
FOM. Os dados quanto ao total de espécies da FOM ainda são incompletos. Fazse necessário que levantamentos sejam direcionados aos escassos remanescentes que restam e que o critério amostral estenda-se sobre todas as formas biológicas.

Palavras-chave: floresta com araucária; florística; levantamento qualitativo e vegetação.

\section{Abstract}

A floristic inventory of woody species was made in an area of 41 ha of Mixed Ombrophylous Forest (FOM) of Araucarias Municipal Park located in Guarapuava PR. The area has the following geographic coordinates $25^{\circ} 20^{\prime} 44^{\prime \prime}$ and $25^{\circ} 21^{\prime} 35^{\prime \prime}$ South latitude and $51^{\circ} 27$ ' $31^{\prime}$ ” and $51^{\circ} 28$ ' $16^{\prime}$ " West longitude. The altimetric level of the Park is around 1070 meters above sea level, the dominance is gentle undulating topography and soil type Ferrasol acric Humic. The survey was conducted from Mar/04 to Oct/08 with an average of one visit every 20 days. It was identified 107 species, 77 genera and 41 families distributed in biological forms of microphanerophyts $(n=44)$ mesofanerophytes $(n=39)$, nanophanerophytes (n $=15)$, lianas $(n=8)$ and megafanerophyte $(n=1)$. The families with the highest diversity were 12 species in Solanaceae, Myrtaceae with nine, eight Fabaceae, Bignoniaceae with six, five Asteraceae. These families were grouped $37.4 \%$ of the species, while the other 38 families accounted for $62.6 \%$ of diversity. Approximately $60.5 \%$ of families are represented by only one species each. Considering the criteria frequency Allophylus edulis (A. St.-Hil., A.Juss \& Cambess.) Hieron. ex Niederl., Casearia decandra Jacq., Cedrela fissilis Vell., Cinnamodendron dinisii Schwacke, Jacaranda puberula Cham., Matayba elaeagnoides Radlk., Ocotea porosa (Nees \& Mart.) Barroso, Ocotea puberula (Rich.) Nees, Ocotea pulchella (Nees) Mez, Schinus terebinthifolius Raddi, Vernonanthura discolor (Spreng.) H. Rob. and Zanthoxylum rhoifolium Lam. species can be considered mates from Araucaria angustifolia in Flora of FOM. Data about the total species of the FOM are still incomplete. It is necessary that surveys are directed to the few remnants that remain and that the test sample extends over all biological forms.

Key words: araucaria forest; flora; vegetation and qualitative survey.

\section{Introdução}

Do ponto de vista nomenclatural, a presença da Araucaria angustifolia, Pinheiro brasileiro, Pinheiro-do-Paraná ou simplesmente pinheiro caracteriza um tipo de formação vegetal que ao longo dos tempos recebeu nomes como araucarietum, araucarilândia, floresta aciculifoliada, floresta com araucária, floresta de araucária, floresta pinatifoliada, floresta de pinheiros, formação araucária, mata de araucária, matas de pinhais, matas mistas, matas pretas, pinhal, pinheiral, zona dos pinhais ou floresta ombrófila mista (FOM), sendo este último nome o mais usado atualmente (MAACK, 1948; KLEIN, 1960; 
VELOSO, 1962; REITZ e KLEIN, 1966; ROMARIZ, 1972; BACKES e NILSON, 1983; VELOSO et al., 1991; IBGE, 1992; LEITE, 2002; RODERJAN et al., 2002).

Entre as formações vegetacionais brasileiras, Veloso et al. (1991) colocam que a FOM torna-se fisionomicamente inconfundível pela presença da Araucaria angustifolia (Bertol.) Kuntze (Araucariaceae). Segundo IBGE (1992), a FOM é caracterizada como vegetação caracterizadora do Planalto Meridional, encontrada atualmente em disjunções florísticas em refúgios situados nas Serras do Mar e da Mantiqueira. No entendimento de Leite (1995) a definição de Floresta Ombrófila Mista deve-se à mistura de floras de diferentes origens com representantes da flora tropical e temperada em marcada relevância estrutural de elementos Coniferales e Laurales resultando em padrões fitofisionômicos típicos. Para Klein (1960), as FOM estão longe de serem associações homogêneas e contínuas, sendo formadas por muitas associações e agrupamentos que apresentam espécies características e próprias de cada estágio de sucessão.

O conhecimento da composição florística consiste no primeiro passo para a compreensão da real dimensão da biodiversidade local, pois, produz um rol de espécies ocorrentes, sendo que para isso, é de fundamental importância a correta identificação taxonômica dos espécimes encontrados.

O levantamento florístico caracterizase por ser um método qualitativo, cuja metodologia empregada na maioria dos casos é resultante de visitas frequentes aos locais de estudo de maneira a percorrer a maior área possível para coleta de material botânico fértil (BRITEZ et al., 1995; LEONI e TINTE, 2004; CERVI et al., 2007). Pode ser feito também por coletas aleatórias estratificadas previamente definas, ou seja, com coleta de material fértil dos indivíduos pertencentes aos estratos arbóreo, arbustivo, epifítico, herbáceo e lianas (MORO et al., 1996; KOZERA et al., 2006). Alguns estudos realizam o levantamento florístico por meio de coletas durante a aplicação da metodologia fitossociológica, tal como o método de quadrantes (MARTINS, 1993; BERTONI e MARTINS, 1987) ou parcelas (SONDA et al., 1999; BRAZ et al., 2004).

No Paraná, os estudos de Occhioni e Hastschbach (1972), Imaguire (1980a e 1980b), Cervi et al. (1989), Silva e Marconi (1990), Rondon Neto et al. (2002), Kozera et al. (2006), entre outros, foram executados em áreas do $1^{\circ}$ planalto. Para o $2^{\circ}$ planalto são encontrados os trabalhos de Carvalho (1980), Galvão et al. (1989), Britez et al. (1995), Negrelle e Leuchtenberger (2001), Seger et al. (2005), Schaaf et al. (2006), Cervi et al. (2007), entre outros.

Para o $3^{\circ}$ planalto, os estudos são escassos como constatado por Isernhagen (2001) que das 162 referências listadas sobre levantamentos em áreas florestais nas últimas duas décadas no Paraná, apenas uma pertencia a esta região. Nos últimos anos foram realizados os trabalhos de Silva (2004), Watzlawick et al. (2005) e Cordeiro e Rodrigues (2007).

Como as informações sobre a flora desta região são exíguas, este estudo teve como objetivo conhecer as plantas lenhosas de um remanescente florestal localizado no $3^{\circ}$ planalto e comparar a similaridade florística com outras áreas de FOM no Paraná. 
Material e Métodos

O Parque Municipal das Araucárias (PMA) está localizado em Guarapuava
(PR) , entre as coordenadas geográficas $25^{\circ}$ $20^{\prime} 44^{\prime \prime}$ e $25^{\circ} 21^{\prime} 35^{\prime \prime}$ de latitude Sul e $51^{\circ}$ $27^{\prime} 31^{\prime \prime}$ e 51 $28^{\prime} 16^{\prime \prime}$ de Longitude Oeste, (Figura 1). O histórico do Parque revela

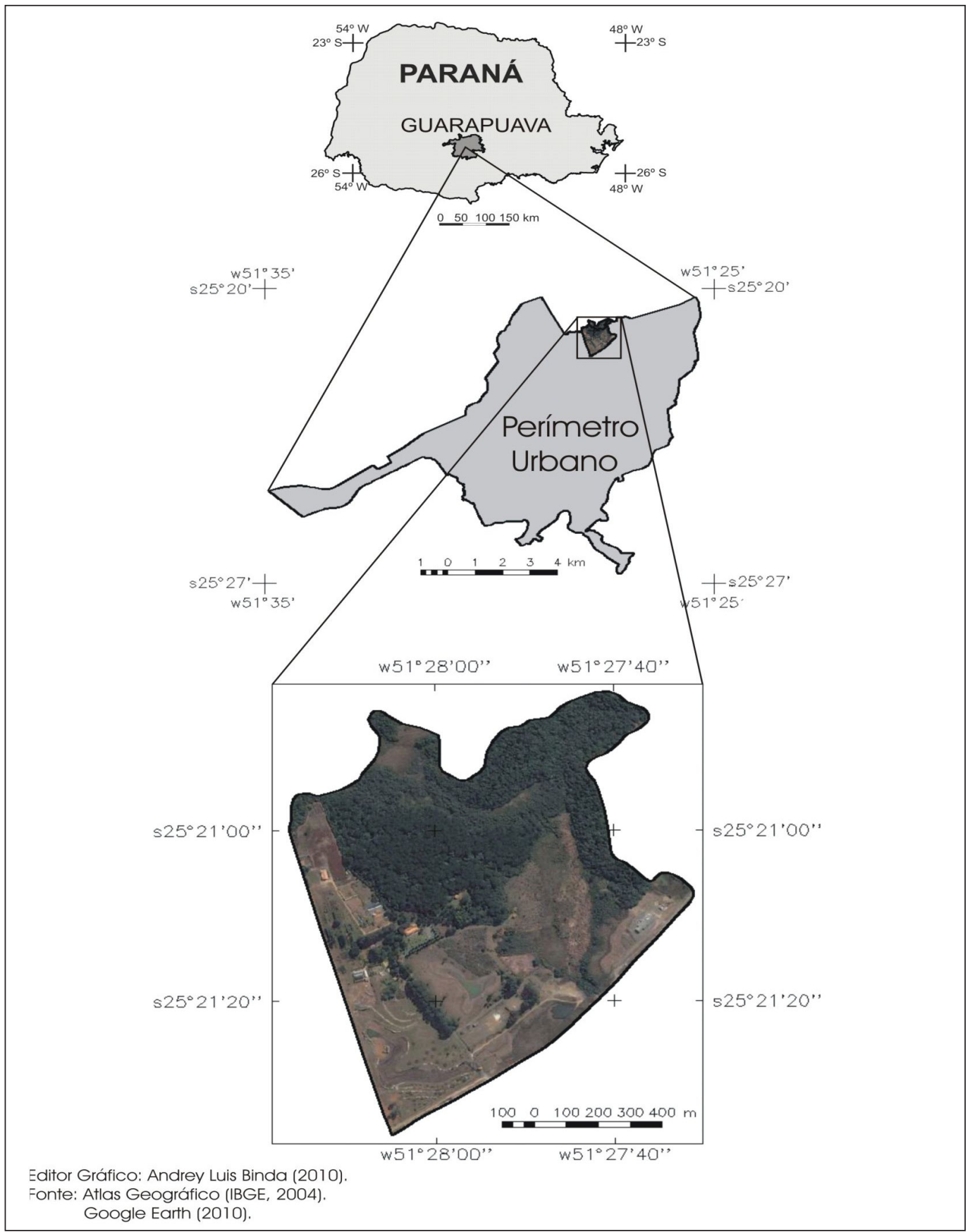

Figura I. Localização do Parque Municipal das Araucárias, Guarapuava, Paraná 
que no passado houve corte seletivo de algumas espécies do subosque para facilitar o manejo de equinos reprodutores que eram criados na área. Contudo, não se verifica sinais de perturbações mais agressivas como desmatamento com a retirada de essências florestais. A área do Parque é de aproximadamente 104 ha, com 41 ha ocupados pela FOM (SEMAFLOR, 2010). A altitude fica em torno dos 1070 m.s.n.m (CORDEIRO; RODRIGUES, 2007).

O clima da região, segundo a classificação de Köppen, é do tipo $\mathrm{Cfb}$, sem estação seca (MAACK, 1981). Os dados climáticos das últimas três décadas, coletados por IAPAR (2010), mostram valores da temperatura média do ar de $17,1^{\circ} \mathrm{C}$, sendo $20,8{ }^{\circ} \mathrm{C}$ a maior média e $12,8^{\circ} \mathrm{C}$ a menor. A umidade relativa do ar variou entre 72 a $81 \%$. Os ventos de maiores velocidades $(3,4$ $\mathrm{m} / \mathrm{s}$ ) foram mais constantes na direção $\mathrm{E}$ e os mais fracos $(2,6 \mathrm{~m} / \mathrm{s})$ no sentido NE. $\mathrm{O}$ índice pluviométrico médio anual foi de $1915 \mathrm{~mm}$, com 92,1 mm para o mês mais seco e $207,8 \mathrm{~mm}$ para o mais chuvoso, e um mínimo de 8 e máximo de 16 dias/mês de chuva. O número de horas de brilho solar variou entre 174,4 a 208,8 h/mês.

O tipo de solo predominante é o Latossolo Bruno Ácrico Húmico LBw (GHIDIN et al., 2006) ocorrendo associações de Latossolo Bruno Álico + Cambissolo Álico e Neossolos Litólicos nas vertentes mais íngremes e na planície colúvio-aluvial Gleissolo Húmico e Orgassolos (RODERJAN et al., 1991). O relevo da área da floresta apresenta três variações bem distintas, suave-ondulado a ondulado na porção superior, médioondulado na porção mediana e plano na porção inferior que margeia o rio Xarquinho.
Olevantamento florístico dos indivíduos lenhosos foi executado empregando-se as técnicas de coleta e herborização propostas por Fidalgo e Bononi (1989). O material coletado foi herborizado, identificado e depositado no Herbário do Departamento de Botânica da Universidade Federal Paraná - UPCB. Entende-se por plantas lenhosas "aquelas que apresentam crescimento secundário, resultante da atividade de dois meristemas laterais - o câmbio vascular e o câmbio da casca, como é o caso de arbustos, árvores e lianas perenes ou cipós lenhosos" (RAVEN et al., 2007).

A ordenação das famílias e gêneros foi baseada Souza e Lorenzi (2008) e a nomenclatura das espécies foi verificada nos arquivos do Missouri Botanical Garden (MOBOT, 2011). O enquadramento das formas biológicas seguiu a classificação de Raunkier adaptada por Matteucci e Colma (1982) sendo considerados os seguintes valores para a altura dos indivíduos lenhosos encontrados a campo: nanofanerófitos $<2 \mathrm{~m}$, microfanerófitos entre 2 e $8 \mathrm{~m}$, mesofanerófitos $>8 \mathrm{e}<30 \mathrm{~m}$, megafanerófitos $\geq 30 \mathrm{~m}$ e lianas lenhosas.

\section{Resultados e Discussão}

O resultado do levantamento florístico foi o registro de 107 espécies de plantas lenhosas pertencentes a 77 gêneros e 41 famílias botânicas (Tabela 1). Destas uma espécie é pertencente à divisão Conipherophyta e 106 à divisão Magnoliophyta. Em relação às formas biológicas (Figura 2), foram identificados 44 microfanerófitos (41,1\% do total), 39 mesofanerófitos (36,4\%), 15 nanofanerófitos (14\%), 8 lianas (7,6\%) e 1 megafanerófito $(0,9 \%)$

As famílias que apresentaram maior diversidade (Figura 3) foram: Solanaceae com 
12 espécies, Myrtaceae com nove, Fabaceae com oito, Bignoniaceae com seis, Asteraceae com cinco. Estas cinco famílias (11,6\%) agruparam 40 espécies $(37,4 \%)$ enquanto que as outras 38 famílias $(88,4 \%)$ responderam por $62,6 \%$ da riqueza florística. Do total de famílias aproximadamente $60,5 \%(n=26)$ estão representadas por apenas uma espécie cada.

Tabela I. Relação de espécies lenhosas do remanescente de Floresta Ombrófila Mista do Parque Municipal das Araucárias, Guarapuava - PR, organizada por famílias, forma biológica e ocorrência em outros estudos

\begin{tabular}{|c|c|c|c|}
\hline FAMÍLIA / ESPÉCIE ${ }^{1}$ & FB $^{2}$ & OCORRÊNCIA ${ }^{3}$ & $\mathbf{N}^{\circ}$ COLETOR \\
\hline \multicolumn{4}{|l|}{ Anacardiaceae } \\
\hline 1. Lithraea aroeirinha March. ex Warm. & Mi & $1,8,14,20,22,23,31$ & J. Cordeiro 020 \\
\hline 2. Schinus johnstonii F. A. Barkley & Mi & 25 & J. Cordeiro 006 \\
\hline 3. Schinus terebinthifolius Raddi & Mi & $\begin{array}{c}1,2,3,4,6,7,8,9,10,12,13, \\
14,17,19,20,21,22,23,24, \\
25,29,30,31\end{array}$ & J. Cordeiro 019 \\
\hline
\end{tabular}

\section{Aquifoliaceae}

4. Ilex brevicuspis Reissek

$\mathrm{Me} \quad 1,12,23,25,31$

J. Cordeiro 248

5. Ilex paraguariensis A. St.-Hil.

$\mathrm{Me} \quad 1,2,3,4,6,8,9,12,13,17,20$, $21,22,23,25,28,31$

J. Cordeiro 123

6. Ilex theezans Mart. ex Reissek

Me $\quad 1,2,3,4,8,9,12,14,20,21$,
$\quad 22,23,24,27,28,29,30,31$

J. Cordeiro 291

\section{Araucariaceae}

7. Araucaria angustifolia (Bertol.)

Kuntze

$1,2,3,4,6,7,8,9,10,12,13$,

Ma 14, 15, 17, 19, 20, 21, 22, 23, J. Cordeiro 014 $24,25,28,29,30,31$

\section{Asteraceae}

8. Baccharis dentata (Vell.) G.M.

Barroso

$\begin{array}{lll}\text { Mi } & 31 & \text { J. Cordeiro } 027\end{array}$

9. Baccharis semiserrata DC. var.

elaeagnoides (Steud. ex. Baker) G. M

Barroso

10. Gochnatia polymorpha (Less.)

Cabrera

11. Pitptocarpha cf. angustifolia

(Spreng.) H. Rob

12. Vernonanthura discolor (Less.) H.

Robinson

\section{Berberidaceae}

13. Berberis laurina Billb.

\section{Bignoniaceae}

14. Arrabidaea cf. chica (Humb. \&

Bonpl.) B. Verl.

15. Arrabidaea corallina (Jacq.)

Sandwith

16. Jacaranda puberula Cham.

17 Macfadyena ungis-cati (L.) A. H. Gentry

$\begin{array}{lll}\mathrm{Mi} & \text { 23,25 Cordeiro } 008\end{array}$

Me 1, 2, 3, 8, 13, 17, 23, 24, 25, $31 \quad$ J. Cordeiro 120

$\mathrm{Me} 3,4,8,9,12,13,14,15,20,22$, J. Cordeiro s/n Me $\begin{array}{r}1,3,4,8,9,10,12,13,14,15, \quad \text { J. Cordeiro } 028 \\ 17,20,21,23,24,25,27,31\end{array}$

$\mathrm{Na} \quad 12,20,22,24,25 \quad$ J. Cordeiro 012

$\begin{array}{lll}\mathrm{Li} & - & \text { J. Cordeiro } 305\end{array}$

Li - $\quad$ J. Cordeiro 151

Me 2, 3, 6, 7, 8, 9, 10, 12, 13, 14, J. Cordeiro 026

Li 10,12,21,22 J. Cordeiro 302

(continua...) 
(continuação...)

\begin{tabular}{lccc}
\hline FAMÍLIA / ESPÉCIE & FB $^{2}$ & OCORRÊNCIA $^{3}$ & N $^{\circ}$ COLETOR \\
\hline $\begin{array}{l}\text { 18. Pithecoctenium crucigerum (L.) A. H. } \\
\text { Gentry }\end{array}$ & $\mathrm{Li}$ & 7,25 & J. Cordeiro 153 \\
$\begin{array}{l}\text { 19. Handroanthus albus (Cham.) } \\
\text { Mattos } \quad \mathrm{Me}\end{array}$ & $23,24,31$ & J. Cordeiro 306 \\
$\begin{array}{l}\text { Cannabaceae } \\
\text { 20. Celtis iguanaea (Jacq.) Sarg. }\end{array}$ & $\mathrm{Mi}$ & 29 & J. Cordeiro 112
\end{tabular}

\section{Cannelaceae}

21. Cinnamodendron dinisii Schwacke

\section{Cardiopteridaceae}

22. Citronella gongonha (Mart.) R.A. Howard

\section{Caricaceae}

23. Carica quercifolia (A. St.-Hil.)

Hieron.

\section{Celastraceae}

24. Maytenus muelleri Schwacke

\section{Clethraceae}

25. Clethra scabra Pers.

\section{Cunoniaceae}

26. Lamanonia speciosa (Cambess.) L. B. Sm.

\section{Erythroxylaceae}

27. Erytbroxylum deciduum A. St.-Hil. Mi

\section{Escalloniaceae}

28. Escallonia bifida Link \& Otto

$\mathrm{Mi}$

\section{Euphorbiaceae}

29. Bernardia pulchella (Baill.) Müll. Arg. Mi

30. Sapium glandulosum (L.) Morong Me

31. Sebastiania brasiliensis Spreng. Mi

32. Sebastiania commersoniana (Baill.)

L.B. Sm. \& Downs

\section{Fabaceae}

33. Senegalia recurva (Benth.) Siegler \& Ebinger

34. Canalavia bonariensis Lindl.

35. Dalbergia frutescens (Vell.) Britton

36. Erythrina crista-galli L.

$\mathrm{Me}$

$\mathrm{Mi}$

37. Erythrina sp

$\mathrm{Me}$

38. Mimosa pilulifera var. pseudincana

(Burkart) Barneby

$\mathrm{Mi}$

$1,2,3,4,6,8,9,10,12,13,14, \quad$ J. Cordeiro 021
$19,20,21,22,23,25,30,31$

$\begin{array}{cc}12 & \text { J. Cordeiro } 142 \\ 12,17,19,20,22,25,27,31 & \text { J. Cordeiro } 126 \\ 10,21,22,23,31 & \text { J. Cordeiro s/n } \\ - & \text { J. Cordeiro s/n } \\ - & \text { J. Cordeiro } 003\end{array}$


(continuação...)

FAMÍLIA / ESPÉCIE ${ }^{1}$
39. Mimosa scabrella Benth.
40. Senna araucarietorum H.S. Irwin \&
Barneby Lamiaceae

41. Vitex megapotamica (Spreng.)

Moldenke

Me $\quad 3,6,8,10,12,14,17,19,20$
$22,23,25,30,31$ $\mathbf{N}^{\circ}$ COLETOR

$\begin{array}{ccc}\text { FB }^{2} & \text { OCORRENCIA }^{3} & \mathbf{N}^{\circ} \text { COLETOR } \\ \mathrm{Me} & 1,2,3,6,8,10,13,14,21,22, & \text { J. Cordeiro } 272 \\ \mathrm{Mi} & 23,24,25,28,31 & \text { J. Cordeiro } 128\end{array}$

\section{Lauraceae}

42. Cinnamomum amoenum (Nees)

Kosterm.

$\mathrm{Me}$

25,30

$1,2,3,4,7,8,9,12,13,14,15$, $20,21,22,23,24,25,27,28,31$ $1,2,3,4,6,7,8,9,10,12,14$

44. Ocotea puberula (Rich.) Nees

Me $15,17,20,21,22,23,24,25$, $27,28,31$
$1,3,6,7,12,14,19,20,21,22$,
$24,25,27,28,30,31$

45. Ocotea pulchella (Nees) Mez

J. Cordeiro 106

43. Ocotea porosa (Nees \& Mart.) Barroso

\section{Loganiaceae}

46. Strychnos brasiliensis (Spreng.) Mart. Mi

$12,19,20,23,25,28$

J. Cordeiro 099

J. Cordeiro 101

J. Cordeiro 267

$\mathrm{Me} \quad \begin{gathered}1,3,6,7,12,14,19,20,21,22 \\ 24,25,27,28,30,31\end{gathered}$

J. Cordeiro 001

\section{Lythraceae}

47.Heimia myrtifolia Cham. et Schltdl. Na

\section{Malvaceae}

48. Luehea divaricata Mart.

$\mathrm{Me}$

\section{Melastomataceae}

49. Leandra xanthocoma (Naudin) Cogn. $\mathrm{Na}$

$\mathrm{Mi}$

50. Miconia cineracens Miq.

$\mathrm{Na}$

51. Miconia byemalis A. St.-Hil. \&

Naudin

\section{Meliaceae}

52. Cedrela fissilis Vell.

\section{Myrsinaceae}

53. Myrsine coriacea (Sw.) R. Br. ex Roem. \& Schult.

$$
3,22,25
$$

J. Cordeiro 133

$2,3,4,6,8,9,10,12,13,15$, $17,19,22,23,25,30,31$

J. Cordeiro 307

$$
12,21,25
$$

J. Cordeiro 121

$$
19,22,23,24,25,30,31
$$

J. Cordeiro 125

$$
3,6,22,24,25,31
$$

J. Cordeiro 250

$\mathrm{Me}$

$1,2,3,4,8,10,12,13,14,15$, $17,20,21,23,25,27,31$

J. Cordeiro 308

$\mathrm{Me}$

$2,3,8,10,12,13,14,20,21$, $22,25,28,30,31$

J. Cordeiro 251

\section{Myrtaceae}

54. Calyptranthes concinna DC.

$\mathrm{Mi}$

$3,12,17,19,20,21,22,24,25$,

$$
28,30,31
$$

Me

(Cambess.) O. Berg

$12,13,14,20,31$

J. Cordeiro 135

$\mathrm{Me}$

$2,3,6,8,9,10,12,13,14,17$,

57. Eugenia pyriformis Cambess.

$\mathrm{Me}$

58. Eugenia uniflora L. $19,20,21,25,28,29,30,31$

$$
\begin{array}{cc}
23,31 & \text { J. Cordeiro } 105 \\
2,6,7,8,9,10,12,13,14,15, & \text { J. Cordeiro } 310 \\
17,19,20,23,25,31 &
\end{array}
$$$$
19,20,21,25,28,29,30,31 \quad \text { J. Cordeiro } 040
$$

J. Cordeiro 309

J. Cordeiro 040

(continua...) 
(continuação...)

\begin{tabular}{l} 
FAMÍLIA / ESPÉCIE ${ }^{1}$ \\
\hline 59. Eugenia uruguayensis Cambess. \\
60. Myrcia guianensis (Aubl.) DC. \\
61. Myrcia hartwegiana (O. Berg.) \\
Kiaersk. \\
62. Myrcia venulosa DC. \\
$\quad$ Oleaceae \\
63. Ligustrum lucidum W.T. Aiton \\
exótica \\
64. Ligustrum sinense Lour. exótica \\
$\quad$ Phytolacaceae \\
65. Phytolacca dioica L. \\
$\quad$ Proteaceae \\
66. Roupala brasiliensis Klotzsch \\
$\quad$ Rhamnaceae \\
67. Hovenia dulcis Thunb. exótica \\
$\quad$ Rosaceae \\
68. Eriobotrya japonica (Thunb.) Lindl. \\
exótica \\
69. Prunus sellowii Koehne \\
70. Rubus brasiliensis Mart. \\
71. Rubus erytbrocladus Mart.
\end{tabular}

$\begin{array}{cc}\text { FB }^{2} & \text { OCORRENCIA }^{3} \\ \mathrm{Mi} & 19,21,31 \\ \mathrm{Me} & 21 \\ \mathrm{Mi} & 30\end{array}$

$\mathrm{N}^{\circ}$ COLETOR

$\mathrm{Mi}$

17, 22, 25, $31 \quad$ J. Cordeiro 312

J. Cordeiro 311

J. Cordeiro 038

J. Cordeiro 131

Mi

10,31

J. Cordeiro 050

$\mathrm{Mi}$

J. Cordeiro 049

$\mathrm{Me}$

31

J. Cordeiro 089

Me 3, 4, 6, 7, 8, 9, 12, 13, 15, 22, J. Cordeiro 313

$$
23,25,27,31
$$

$\mathrm{Me}$

$13,19,25,31$

J. Cordeiro 264

Mi

$10,13,25,31$

J. Cordeiro 232

Me 2, 3, 4, 6, 7, 8, 9, 12, 13, 15, 17, $19,21,25,27,28,30,31$

J. Cordeiro 048

$\mathrm{Li}$

$12,22,23,25$

J. Cordeiro 118

$\mathrm{Na}$

$12,22,25$

J. Cordeiro 032

\section{Rubiaceae}

72 Alibertia concolor (Clam.) K. Schum. Na

$3,12,17,25,31$

J. Cordeiro 167

73. Guettarda uruguensis Cham. \&

Schltdl.

$\mathrm{Mi}$

$6,10,12,17,19,22,25,30,31$

J. Cordeiro 314

74. Palicourea marcgravii A. St.-Hil.

75. Rudgea parquioides (Cham.) Müll.

Arg.

$\mathrm{Na}$

22

J. Cordeiro 140

$\mathrm{Na}$

$3,19,25,30$

J. Cordeiro 037

\section{Rutaceae}

76. Citrus limon (L.) Osbeck exotica

77. Citrus reticulata Blanco exótica

$\mathrm{Mi}$

31

J. Cordeiro 161

$\mathrm{Mi}$

J. Cordeiro 263

78. Citrus sinensis (L.) Osbeck exótica

$\mathrm{Mi}$

-

J. Cordeiro 266

79. Zanthoxylum rhoifolium Lam.

\section{Salicaceae}

80. Banara tomentosa Clos

81. Casearia decandra Jacq.

82. Casearia obliqua Spreng.

Me $\begin{gathered}3,4,6,7,8,9,10,12,13,14,15, \quad \text { J. Cordeiro } 098 \\ 17,20,22,23,25,29,30,31\end{gathered}$

Mi 1, 12, 17, 20, 23, 25, $31 \quad$ J. Cordeiro 129

$3,4,6,7,9,10,12,13,14,17$,

Me 19,20,21, 23, 25, 27, 28, 29, J. Cordeiro 264 30,31

Me 8, 10, 12, 14, 17, 20, 25, 27, $31 \quad$ J. Cordeiro 205

(continua...) 
(Continuação...)

\begin{tabular}{|c|c|c|c|}
\hline FAMÍLIA / ESPÉCIE ${ }^{1}$ & $\mathbf{F B}^{2}$ & OCORRÊECIA ${ }^{3}$ & $\mathrm{~N}^{\circ}$ COLETOR \\
\hline 83. Xylosma ciliatifolia (Clos) Eichler & $\mathrm{Mi}$ & $3,6,10,17,22,25,31$ & J. Cordeiro 301 \\
\hline \multicolumn{4}{|l|}{ Sapindaceae } \\
\hline $\begin{array}{l}\text { 84. Allophylus edulis (A. St.-Hil., A.Juss } \\
\text { \& Cambess.) Hieron. ex Niederl. }\end{array}$ & $\mathrm{Mi}$ & $\begin{array}{c}2,3,4,6,7,8,9,10,12,14,17 \\
19,20,21,23,25,28,29,31\end{array}$ & J. Cordeiro 007 \\
\hline 85. Cupania vernalis Cambess. & $\mathrm{Mi}$ & $\begin{array}{c}6,8,9,12,13,14,17,20,23 \\
24,25,27,28,30,31\end{array}$ & J. Cordeiro 315 \\
\hline 86. Matayba elaeagnoides Radlk. & $\mathrm{Me}$ & $\begin{array}{c}2,3,4,6,8,9,10,12,13,14 \\
17,19,21,22,23,24,25,27 \\
28,30,31\end{array}$ & J. Cordeiro 095 \\
\hline 87. Thinouia mucrona Radlk. & $\mathrm{Li}$ & - & J. Cordeiro 176 \\
\hline \multicolumn{4}{|l|}{ Simaroubaceae } \\
\hline 88. Castela tweediei Planch. & $\mathrm{Mi}$ & - & J. Cordeiro 273 \\
\hline \multicolumn{4}{|l|}{ Solanaceae } \\
\hline 89. Athenaea picta (Mart.) Sendtn. & $\mathrm{Mi}$ & - & J. Cordeiro 041 \\
\hline 90. Brunfelsia pilosa Plowman & $\mathrm{Na}$ & - & J. Cordeiro 013 \\
\hline 91. Cestrum amictum Schltdl. & $\mathrm{Mi}$ & 25,31 & J. Cordeiro 316 \\
\hline 92. Solanum cf. caeruleum Vell. & $\mathrm{Me}$ & - & J. Cordeiro 035 \\
\hline 93. Solanum granuloso-leprosum Dunal & $\mathrm{Mi}$ & $10,20,25,31$ & J. Cordeiro 138 \\
\hline 94. Solanum megalochiton Mart. & $\mathrm{Na}$ & 25 & J. Cordeiro 259 \\
\hline 95. Solanum paranense Dusén & $\mathrm{Na}$ & - & J. Cordeiro 175 \\
\hline 96. Solanum pseudoquina A. St.-Hil. & $\mathrm{Me}$ & $3,14,17,25,31$ & J. Cordeiro 113 \\
\hline 97. Solanum ramulosum Sendtn. & $\mathrm{Na}$ & - & J. Cordeiro 005 \\
\hline 98. Solanum sanctaecatharinae Dunal & $\mathrm{Me}$ & $3,6,7,8,10,12,17,23,25,31$ & J. Cordeiro 033 \\
\hline 99. Solanum variabile Mart. & $\mathrm{Mi}$ & 12,22 & J. Cordeiro 043 \\
\hline 100. Vassobia breviflora (Sendtn.) Hunz. & $\mathrm{Na}$ & $7,20,25,31$ & J. Cordeiro 024 \\
\hline
\end{tabular}

\section{Styracaceae}

101. Styrax leprosus Hook. \& Arn.

\section{Symplocaceae}

102. Symplocos uniflora (Pohl) Benth. Mi

\section{Thymelaeaceae}

103. Daphnopsis racemosa Griseb.

Urticaceae

104. Urera baccifera (L.) Guadich. ex Wedd.

\section{Verbenaceae}

105. Lantana brasiliensis Link

106. Lantana camara L.

$\mathrm{Na} \quad 3,7,19,20,22,24,25,30,31 \quad J$. Cordeiro 030

$\mathrm{Mi}$

$2,3,4,8,9,12,13,15,17,20$ $23,25,28,31$

J. Cordeiro 102

$6,10,12,19,20,21,25,28$ 30,31

J. Cordeiro 025

$\mathrm{Mi}$

$7,10,25,31$

J. Cordeiro 206

J. Cordeiro 134

J. Cordeiro 017

(Continua...) 
(Conclusão)

\begin{tabular}{lccc}
\hline FAMÍLIA / ESPÉCIE & FB $^{2}$ & OCORRÊNCIA $^{3}$ & N $^{\circ}$ COLETOR $^{-}$ \\
\hline Winteraceae & & & \\
& & & \\
$\begin{array}{l}\text { 107. Drimys brasiliensis subsp. sylvatica } \\
\text { (A. St.-Hil.) Ehrend. \& Gottsb. }\end{array}$ & $\mathrm{Mi}$ & $\begin{array}{c}2,3,4,8,12,13,14,20,21,22, \\
23,24,25,31\end{array}$ & J. Cordeiro 022 \\
\hline
\end{tabular}

I $O$ enquadramento das famílias, gêneros e espécies foi baseado no APG II. 2 Para a forma biológica foi considerada a classificação de Raunkier adaptada por Matteucci e Colma, ( 1 982). FF = Forma biológica. Li = Liana; Ma = Macrofanerófito; $\mathrm{Me}=$ Mesofanerófito; $\mathrm{Mi}=$ Microfanerófito; $\mathrm{Na}=$ Nanofarófito. 3 Estudos: I - Occhioni e Hastsbach (1972), 2 - Longhi (1980), 3 - Imaguire (I 980a e 1980b), 4 - Oliveira e Rotta (1982), 6 - Cervi et al. (1987), 7 - Cervi et al. (I 989), 8 Galvão et al. (1989), 9 - Silva e Marconi (1990), 10 - Roseira (1990), I 2 - Britez et al. (1995), 13 - Durigan (1999); Pizatto (1999), I 4 - Ziller (2000), I5 - Negrelle e Leuchtenberger (200I), I 7 - Rondon Neto et al. (2002a), I 9 - Barddal (2004), 20- Silva (2003), 21 - FUPEF (2003), 22 - Cervi et al. (2007), 23 - Carvalho (I 980), 24 - Moro et al., ( 1996), 25 - Kozera et al. (2006), 27 - Sonda et al. (1999), 28 -Watzlawick et al. (2005), 29 - Seger et al. (2005), 30- lurk et al. (2009), 3 I - Isernhagen (200I). exótica - Espécie exótica.

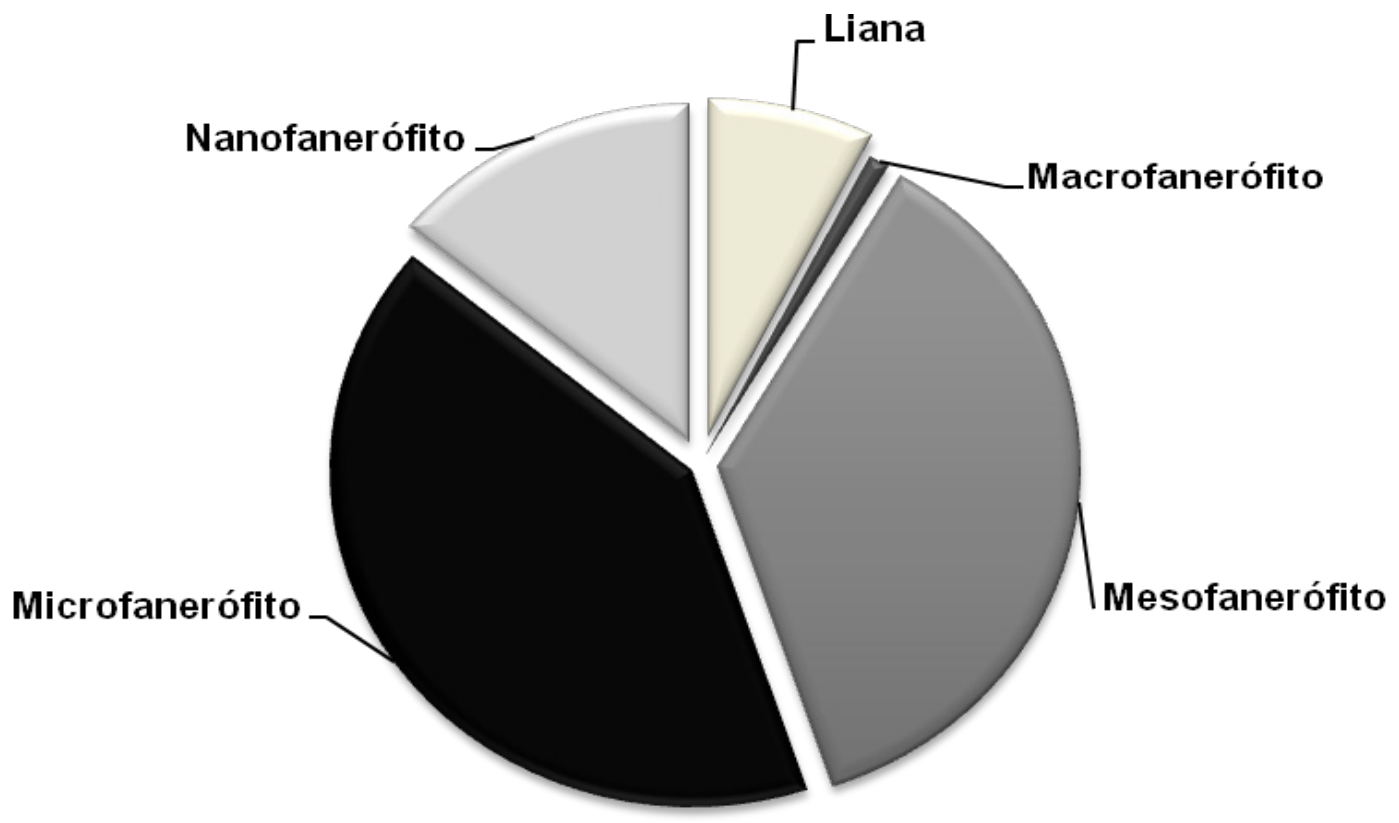

Figura 2. Distribuição das formas biológicas das plantas lenhosas do remanescente de Floresta Ombrófila Mista do Parque Municipal das Araucárias, Guarapuava, Paraná

Considerando o grupo de famílias com maior número de espécies, IBGE (1992) e Leite (1995) destacam que Myrtaceae, Lauraceae e Aquifoliaceae são elementos importantes no conjunto florístico dos ambientes de FOM. Resultados semelhantes foram encontrados em levantamentos executados por Cervi et al. (1987), Roseira (1990) e Curcio et al. (2007) localizados na região do $1^{\circ}$ planalto paranaense, Carvalho (1980), Galvão et al. (1989), Oliveira et al. (2003) e Iurk et al. (2009) no $2^{\circ}$ planalto e FUPEF (2003), Silva (2004) e Watzlawick et al. (2005) no $3^{\circ}$ plantalto. A maior representatividade quanto à riqueza de espécies, vem ratificar a importância ecológica que estas famílias desempenham na composição vegetal dos remanescentes de FOM. 


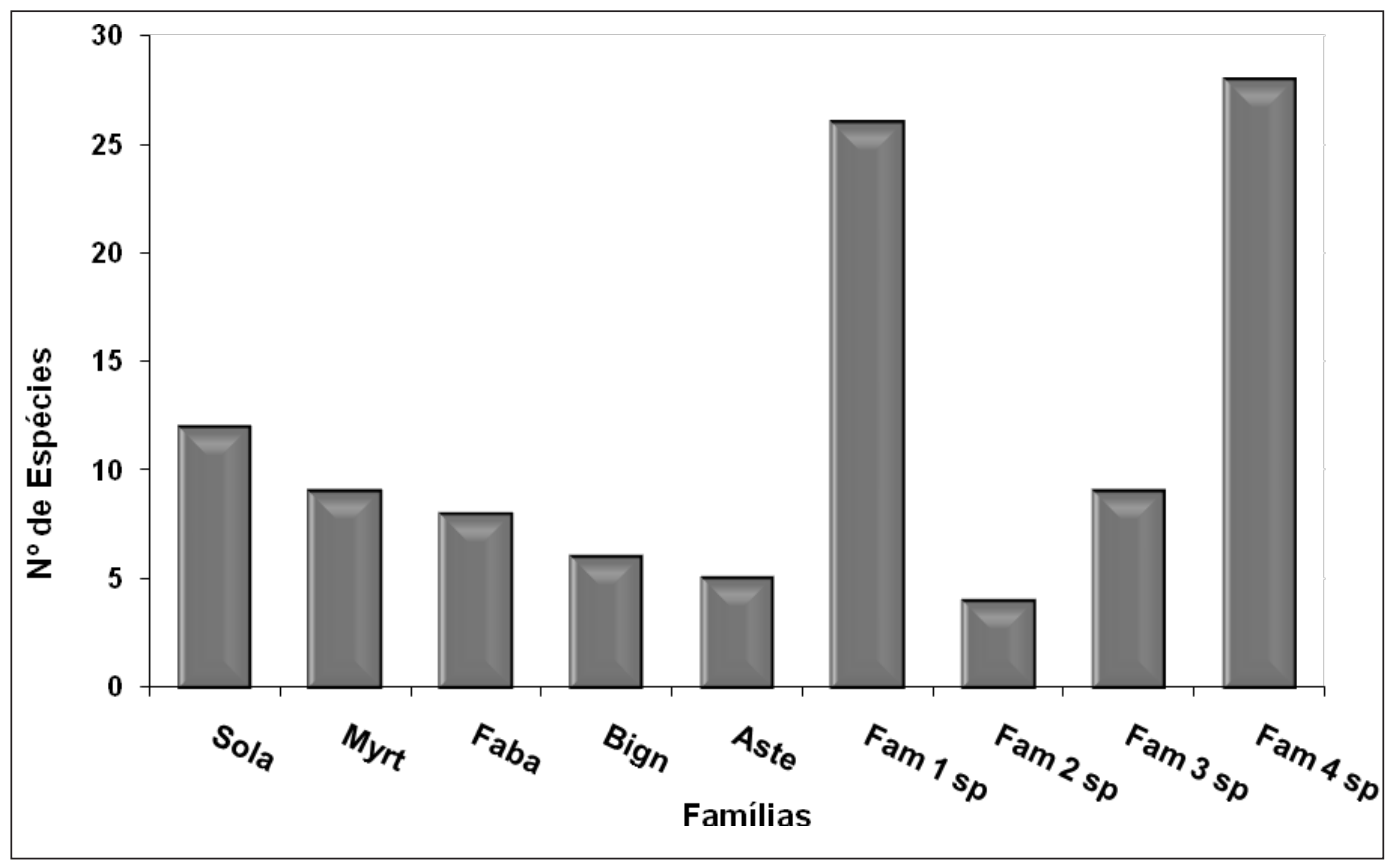

Figura 3. Quantidade de espécies/família encontradas no remanescente de Floresta Ombrófila Mista do Parque Municipal das Araucárias, Guarapuava, Paraná. Sola = Solanaceae, Myrt = Myrtaceae, Faba $=$ Fabaceae, Bign = Bignoniaceae, Aste $=$ Asteraceae, Fam $1 \mathrm{sp}$ = famílias com uma espécies, Fam $2 \mathrm{sp}$ = famílias com duas espécies, Fam $3 \mathrm{sp}$ = famílias com três espécies, Fam 4 sp = famílias com quatro espécies

O remanescente florestal do PMA apresenta considerável diversidade com representantes típicos da FOM como espécies de Lauraceae (RODERJAN et al., 2002), de Myrtaceae (MATTOS, 1972), do gênero primitivo Drimys (VELOSO et al., 1991) além da Araucaria angustifolia. Podocarpus lambertii é citada por Veloso (1962), Klein (1984) e IBGE (1992) como espécie que está associada aos remanescentes de FOM, não foi registrada na área do parque.

Quando se considera o critério frequência, as espécies Allophylus edulis, Casearia decandra, Cedrela fissilis, Cinnamodendron dinisii, Jacaranda puberula, Matayba elaeagnoides, Ocotea porosa, Ocotea puberula, Ocotea pulchella, Schinus terebinthifolius, Vernonanthura discolor e Zanthoxylum rhoifolium podem ser consideradas companheiras da Araucaria angustifolia na composição florística da FOM, uma vez que foram registradas em mais $70 \%$ dos estudos analisados. São espécies encontradas em remanescentes localizados em diversos ambientes, sujeitos a diferentes condições geológicas, geomorfológicas e pedológicas, ao longo dos três planaltos paranaenses. A maioria dessas espécies integra as paisagens da FOM sendo citadas no rol florístico de estudos clássicos como Mattos (1972), Klein (1960), Reitz e Klein (1966) e Maack (1981).

As espécies Arrabidaea cf. chica, Arrabidaea corallina, Athenaea picta, Brunfelsia pilosa, Carica quercifolia, Castella twediei, Mimosa pilulifera var. pseudincana, Senna 
araucarietorum, Solanum cf.caeruleum, Solanum paranense Thinouia mucrona não foram relacionadas em nenhum dos levantamentos como analisados. A inexistência de registros não permite afirmar que essas espécies sejam raras ou endêmicas à região do PMA. Esta ausência deve-se ao critério de inclusão, esforço amostral e objetivo a que se destinavam os estudos. $\mathrm{O}$ enquadramento biológico das 11 espécies mostrou que três são lianas, cinco microfanerófitos, um mesofanerófito, dois nanofanerófitos. A ampliação da coleta sobre as demais formas biológicas pode ajudar no entendimento da composição florística da FOM, uma vez que a maioria dos estudos contempla o estrato arbóreo (micro a macrofanerófito). Contudo, esse motivo sinaliza que as espécies não sejam tão comuns neste tipo de vegetação, uma vez que não foram registradas ou quando foram, apresentam baixa frequência. Por outro lado, salienta-se a escassez de referências sobre estudos específicos da florística individual de lianas, nano e microfanófitos das florestas com araucária.

A comparação entre as listagens florísticas de diversos estudos sofre influência direta do critério e o esforço amostral conferido. O emprego da metodologia fitossociológica por parcelas ou distâncias sofre influência direta do critério de inclusão amostral que varia conforme o objetivo final da pesquisa. Da mesma forma, o esforço amostral fica concentrado apenas na coleta de material dos indivíduos localizados dentro das parcelas ou unidades amostrais. No caso de levantamentos qualitativos, se elege na maioria das vezes uma das formas biológicas como critério de inclusão o que limita o número final de espécies, sendo geralmente, realizados para os indivíduos arbóreos. Neste estudo, como o critério foi a inclusão de todos os indiví- duos lenhosos (de nanofanerófitos a lianas) $\mathrm{e}$ as coletas quinzenais abrangeram a maior extensão do remanescente, a quantidade de espécies (107) foi superior as 58 espécies registradas por Longhi (1980), 67 por Negrelle e Leuchtenberger (2001), 77 por Rondon Neto et al. (2002), 44 por Seger et al. (2005) e 64 por Iurk et al. (2009), entre outros.

Outro fator que influencia a caracterização florística de uma área é a correta identificação das espécies encontradas. Os trabalhos de Roseira (1990), Pizatto (1999), Durigan (1999) e Sonda et al. (1999) possuíam aproximadamente $30 \%$ ou mais das espécies listadas sem identificação positiva. Na lista florística de Oliveira e Rotta (1982) das 103 espécies relacionadas 46,6\% não possuíam o epíteto específico, foram determinadas pelo material vegetativo, o que para algumas plantas pode resultar em erros ou duplicidade taxonômica.

Os estudos de Cervi et al. (2007), Carvalho (1980), Imaguire (1980a e 1980b), Galvão et al. (1989); Britez et al. (1995), Kozera et al. (2006) registraram maior número de espécies comparativamente para a FOM. Essa superioridade deve-se ao fato de que as coletas foram efetuadas em vários ambientes quanto às condições edáficas, em diferentes associações vegetais, como ambiente ciliar, capões, vegetação primária e vegetação secundária (capoeiras).

As espécies de Solanaceae foram coletadas em locais de grande luminosidade como nas margens das trilhas, regiões de encrave da floresta com a Estepe Gramíneo-Lenhosa e clareiras formadas pela queda dos galhos de pinheiros velhos, que evidenciam as características destas plantas de serem heliófilas e pioneiras (LORENZI, 2002). 
A presença e o porte dos caules de oito espécies de lianas lenhosas $(7,5 \%$ da diversidade), principalmente de Arrabidaea corallina, Canavalia bonariensis, Rubus brasiliensis e Thinouia mucrona, pode ser considerado como indicativo que o remanescente encontra-se em estágio avançado de sucessão natural, tomando por base a definição apresentada pela resolução no 31 de 24/08/1998 da Secretaria do Estado do Meio Ambiente (SEMA, 1998). A ocorrência de lianas lenhosas em área que apresentam estágios avançados de sucessão também é citada como sendo uma das principais características dos componentes de estágios seriais proposta por Budowski apud Kuniyoshi (1994).

Como fator negativo destaca-se a presença de sete espécies exóticas (6,5\% do total) como Citrus limon, Citrus reticulata, Citrus sinensis, Eriobotrya japonica, Hovenia dulcis, Ligustrum lucidum e Ligustrum sinense. A forma de invasão destas espécies no sub-bosque da floresta pode ter sido por ação antrópica para Citrus e Eriobotrya japonica, quando o homem dispersa suas sementes após consumir os frutos. Ligustrum e Hovenia dulcis têm a zoocoria como dispersão característica (INSTITUTO HORUS, 2005). Essas plantas podem se tornar invasoras, pois, o processo de invasão começa quando, depois de introduzida em um novo ambiente, a espécie se naturaliza, sendo capaz de se dispersar por grandes áreas, ocasionando graves alterações ao ambiente invadido (CATTANEO, 2005).

Quanto a uma futura invasão, o Ligustrum lucidum oferece maiores riscos, uma vez que dentre as 45 espécies identificadas no levantamento fitossociológico de Cordeiro e Rodrigues (2007) esta espécie obteve o $28^{\circ}$ lugar no valor de importância, fazendose representar na estrutura horizontal do remanescente florestal.

\section{Conclusão}

A composição florística do remanescente florestal do PMA apresentou a ocorrência de espécies típicas desta formação vegetacional. A comparação entre os estudos revelou que para caracterizar a flora de uma área, é fundamental que se realize visitas regulares e abrangentes ao local para a coleta de material fértil e que a listagem final das espécies contenha o nome das plantas identificadas até o epíteto específico.

A FOM mesmo sendo considerada a formação vegetacional peculiar do Paraná não possui registros exatos sobre o total de espécies que compõem a sua florística. Os estudos existentes se desenvolveram principalmente sobre o estrato arbóreo e ficaram concentrados nas regióes do primeiro e segundo planalto paranaense. Faz-se necessário que levantamentos sejam direcionados para a flora dos escassos remanescentes que ainda restam em outras regiões e que o critério amostral estenda-se sobre as demais formas biológicas.

\section{Referências}

BACKES, A.; NILSON, A. D. Araucaria angustifolia (Bert.) O. Kuntze, o pinheiro brasileiro. Iheringia, Porto Alegre, n.30, p.85-96, mar. 1983. 
BARDDAL, M. L.; RODERJAN, C. V.; GALVÃO, F.; CURCIO, G. R. Caracterização florística e fitossociológica de um trecho sazonalmente inundável de floresta aluvial, em Araucária, PR. Ciência Florestal, Santa Maria, v.14. n.2, p.37-50, 2004.

BERTONI,J. E. de A.; MARTINS, F. R. Composição florística de uma floresta na Reserva Estadual de Porto Ferreira, SP. Acta Botanica Brasilica, São Paulo, v.1, n.1, p.17-26, 1987.

BRAZ,D.M.; MOURA,M.V.L.P.; ROSA,M.M.T. Chave de identificação para as espécies de Dicotiledôneas arbóreas da Reserva Biológica do Tinguá, RJ, com base em caracteres vegetativos. Acta Botanica Brasilica, São Paulo, v.18, n2, p.225-240, 2004.

BRITEZ, R.M.; SILVA, S. M.; SOUZA, W. S. de; MOTTA,J.T.W. Levantamento florístico em floresta ombrófila mista, São Mateus do Sul, Paraná, Brasil. Arquivo de Biologia e Tecnologia, Curitiba, v.4, n.38, p.1147-1161, 1995.

CARVALHO, P. E. R. Levantamento florístico da região de Irati - PR (1ª aproximação). Circular Técnica-EMBRAPA, Curitiba, n.3, p.1-44, 1980.

CATTANEO, M. La dispersión de coniferas exóticas en areas naturales: ejemplos de Nueva Zelanda. 2005. Disponível em: < http://www.institutohorus.org.br/download. htm\#artigocien. Acesso em: 15 jul. 2005.

CERVI, A. C.; SCHIMMELPFENG, L. C. T.; PASSOS, M. Levantamento do estrato arbóreo do capão da Educação Física da Universidade Federal do Paraná Curitiba - Paraná - Brasil. Estudos de Biologia, Curitiba, n. 17, p. 49 - 61, ago, 1987.

CERVI, A. C.; PACIORNIK, E. F.; VIEIRA, R. F.; MARQUES, L. C. Espécies vegetais de um remanescente de floresta de araucária (Curitiba, Brasil): Estudo Preliminar I. Acta Biológica Paranaense, Curitiba, v.1/2/3/4, n.18, p.73-114, 1989.

CERVI, A. C.; LINSINGEN, L. V.; HATSCHBACH, G.; RIBAS, O. S. A vegetação do Parque Estadual de Vila Velha, Município de Ponta Grossa, Paraná, Brasil. Boletim do Museu Botânico Municipal, Curitiba, n. 69, 2007.

CORDEIRO, J.; RODRIGUES, W. A. Caracterização fitossociológica de um remanescente de Floresta Ombrófila Mista em Guarapuava, PR. Revista Árvore, Viçosa, v.31, n.3, p.545$554,2007$.

CURCIO, G. R.; GALVÃO, F.; BONNET, A.; BARDDAL, M. L.; DEDECEK, R. A. A floresta fluvial em dois compartimentos do Rio Iguaçu, Paraná, Brasil. Revista Floresta, Curitiba, v.37, n. 2, p. 125-146, mai/ago. 2007.

DURIGAN, M. E. Florística, dinâmica e análise protéica de uma Floresta Ombrófila Mista em São João do Triunfo - PR. 1999. 83 f. Dissertação (Mestrado em Engenharia Florestal) - Setor de Ciências Agrárias, Universidade Federal do Paraná, Curitiba, 1999. 
FIDALGO, O.; BONINI, V. L. R. Técnicas de coleta, preservação e herborização de material botânico. São Paulo: Instituto de Botânica, 1989.

FUNDAÇÃO DE PESQUISAS FLORESTAIS DO PARANÁ - FUPEF. Diagnóstico da Cobertura Vegetal da Área Proposta para a Construção da PCH São Jerônimo e do Contexto Vegetacional do seu entorno. Curitiba: BRASCAN/FUPEF, 2003.

GALVÃO, F.; KUNIYOSHI, Y. S.; RODERJAN, C. V. Levantamento fitossociológico das principais associações arbóreas da Floresta Nacional de Irati - PR. Revista Floresta, Curitiba, v. 19, n.1/2, p.30-49, 1989.

GHIDIN, A. A.; MELO, V. de F.; LIMA, V. C.; LIMA, J. M. J. C. Topossequências de Latossolos originados de rochas basálticas no Paraná: I - mineralogia da fração argila. Revista Brasileira de Ciência do Solo, Viçosa , v. 30, n. 2, abr. 2006. Disponível em: <http://www. scielo.br/scielo.php?script $=$ sci_arttext\&pid=S0100-06832006000200010\&lng=pt\&nrm=i so>. Acesso em: 20 jul. 2009.

GOOGLE EARTH. Imagem de satélite do Parque Municipal das Araucárias. Disponível em: <htpp://www.googleearth.com.br/>. Acesso em: 7 jul. 2011. (Data da imagem: 14 Jun.

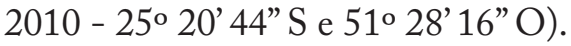

INSTITUTO AGRONÔMICO DO PARANÁ - IAPAR -Cartas climáticas - médias históricas. Disponível em: <http://www.iapar.br/arquivos/Image/monitoramento/medias_ Historicas/Guarapuava.htm>. Acesso em: 15 jul. 2010.

IMAGUIRE, N. Contribuição ao estudo florístico e ecológico da Fazenda Experimental do Setor de Ciências Agrárias da Universidade Federal do Paraná. 1 - Ecologia, origem e ecese da vegetação. Acta Biológica Paranaense, Curitiba, v. 8/9, p. 19-45, 1980a.

IMAGUIRE, N. Contribuição ao estudo florístico e ecológico da Fazenda Experimental do Setor de Ciências Agrárias da Universidade Federal do Paraná. 2- O porque da existência dos campos e matas no primeiro e segundo planaltos paranaenses. Acta Biológica Paranaense, Curitiba, v. 8/9, p. 42-72, 1980b.

INSTITUTO BRASILEIRO DE GEOGRAFIA E ESTATÍSTICA - IBGE. Manual técnico davegetação brasileira. Série - Manuais técnicos em geociências, n.1. Rio de Janeiro: IBGE, 1992.

INSTITUTO BRASILEIRO DE GEOGRAFIA E ESTATÍSTICA - IBGE. Atlas geográfico. Rio de Janeiro: FAE. 2004. 1 CD-ROM

INSTITUTO HORUS. Trabalhos em andamentos - Espécies exóticas e invasoras: fichas técnicas. 2005. Disponível em: < http://www.institutohorus.org.br/trabalhosa_fichas.htm. Acesso em: 15 jul. 2005.

ISERNHAGEN, I.A Fitossociologia Florestal no Paraná e os programas de Recuperação de Áreas Degradadas: uma Avaliação. 2001. 134 f. Dissertação (Mestrado em Botânica) - Setor de Ciências Biológicas, Universidade Federal do Paraná, Curitiba, 2001. 
IURK, M. C.; SANTOS, E. P. dos; DLUGOSZ, L. F.; TARDIVO, R. C. Levantamento florístico de um fragmento de floresta ombrófila mista aluvial do rio Iguaçu, município de Palmeira (PR). Revista Floresta, Curitiba, v.39, n. 3, p.605-615, 2009.

KLEIN, R. M. O aspecto dinâmico do pinheiro brasileiro. Selowia, Itajaí, n. 12, p. 17- 44, 1960.

KLEIN, R. M. Aspectos dinâmicos da vegetação do sul do Brasil. Selowia, Itajaí, n.36, p.5-54, 1984.

KOZERA, K.; DITTRICH, V. A. de O.; SILVA, M. S. Composição florística da floresta ombrófila mista montana do Parque Municipal do Barigui, Curitiba, PR. Revista Floresta, Curitiba, v. 36, n.1, jan./abr. p.45-58. 2006.

KUNIYOSHI, Y. S. Reconhecimento de fases sucessionais de vegetação arbórea. In: A vegetação natural do Estado do Paraná. Curitiba: IPARDES, CTD. 1994.

LEITE, P. F. As diferentes unidades fitoecológicas da região Sul do Brasil - Proposta de Classificação. Cad. Geoc., Rio de Janeiro, n. 15, p. 73 - 164, jul./set. 1995.

LEITE, P. F. Contribuição ao conhecimento fitoecológico do Sul do Brasil. Ciência \& Ambiente. Santa Maria, n.24, p.51-73, jan./jul. 2002.

LEONI, L. de S.; TINTE, V. A. Flora do Parque Estadual da Serra do Brigadeiro: caracterização da vegetação e lista preliminar das espécies. Carangola: Gráfica São José, 2004. 91p.

LONGHI, S. J. A estrutura de uma floresta natural de Araucaria angustifolia (Bertol.) O. Ktze. No sul do Brasil. 1980. 198 f. Dissertação (Mestrado em Ciências Florestais) - Setor de Ciências Agrárias, Universidade Federal do Paraná, Curitiba, 1980.

LORENZI, H.Árvores do Brasil - Manual de Identificação e Cultivo de Plantas Arbóreas do Brasil. vol. 2. 2 ed. Nova Odessa : Instituto Plantarum, 2002.384 p.

MAACK, R. Notas preliminares sobre clima, solos e vegetação do estado do Paraná. Arquivo de Biologia e Tecnologia, Curitiba, v.3, p.103-69. 1948.

MAACK, R. Geografia física do Estado do Paraná. 2. ed. Rio de Janeiro: José Olympio / Sec. da cultura e do esporte do Governo do Estado do Paraná, 1981. 450 p.

MARTINS, F. R. Estrutura de uma floresta mesófila. 2. ed. Campinas: Editora da UNICAMP, 1993.246 p.

MATTEUCCI, S. D.; COLMA, A. Metodologia para el estudo de la vegetacion. Washington, OEA/PRDECT, 1982.168 p.

MATTOS, J. R. O pinheiro brasileiro. 2. ed. São Paulo: José Olympio, 1972. 450 p. 
MORO, R. S.; ROCHA, C. H.; TAKEDA, I. J. M.; KACZMARECH, R. Análise da vegetação nativa da Bacia do Rio São Jorge. Publicatio UEPG - Ciências Biológicas e da Saúde. Ponta Grossa, v.2, n.1, p.33-56, 1996.

MISSOURI BOTANICAL GARDEN - MOBOT. The plant list, 2011. St. Louis. Disponível em: <http://www.tropicos.org/Home.aspx>. Acesso em: 1 maio 2011.

NEGRELLE, R.A.B.; LEUCHTENBERGER, R. Composição e estrutura do componente arbóreo de um remanescente de Floresta Ombrófila Mista. Revista Floresta, Curitiba, v.1 e 2, n.31, p.42-51, 2001.

OCCHIONI, P.; HATSCHBACH, G. A vegetação arbórea dos ervais do Paraná. Leandra, Rio de Janeiro, n.3, p.5-59, dez.1972.

OLIVEIRA, Y. M. M.; ROTTA, E. Levantamento da estrutura horizontal de uma mata de Araucária do primeiro planalto paranaense. Boletim de Pesquisa Florestal, Colombo, n.4, p.1-45, 1982.

OLIVEIRA, E.A. de; RODERJAN, C. V.; CURCIO, R. G.; SILVA, S. M. Caracterização florística, fitossociológica e pedológica de um trecho de floresta ripária dos Campos Gerais do Paraná. Cadernos da Biodiversidade, Curitiba, v.4, n.1, p.8-25, jan. 2003.

PIZATTO, W. Avaliação biométrica da estrutura e da dinâmica de uma floresta ombrófila mista em São João do Triunfo - Pão do Triunfo - P: 1995 a 1998. 1999.172 f. Dissertação (Mestrado em Ciências Florestais) - Setor de Ciências Agrárias, Universidade Federal do Paraná, Curitiba, 1999.

RAVEN, P. H., EVERT R. F.; EICHHORN, S. E. Biologia Vegetal. 7. ed. São Paulo: Editora Guanabara/Koogan, 2007.

REITZ, P. R.; KLEIN, R. M. Araucariáceas. In: REITZ, R. (Ed.). Flora Ilustrada Catarinense. Itajaí: Herbário Barbosa Rodrigues, 1966. p. 1-65.

RODERJAN. C. V.; MILANO, M. S.; FIRKOWSKI, C. Plano de Manejo do Parque Municipal das Araucárias. Guarapuava: SEMAFLOR, 1991.

RODERJAN, C. V.; GALVÃO, F.; KUNIYOSHI, Y. S.; HATSCHBACH, G. G. As unidades fitogeográficas do estado do Paraná. Ciência \& Ambiente, Santa Maria - RS, n.24, p.75-92, jan./jun. 2002.

ROMARIZ, D. de A. A vegetação. In: AZEVEDO, A. de. Brasil - A Terra e o Homem. As Bases Físicas. 2. ed. São Paulo: Companhia Editora Nacional, 1972.v.1, p.521-48.

RONDON NETO, R. M. KOZERA, C.; ANDRADE, R. R.; CECY, A T.; HUMMES, A. P.; FRITZSONS, E.; CALDEIRA, M. V. W.; MACIEL, M. N. M.; SOUZA, M. K. F. Caracterização florística e estrutural de um fragmento de floresta ombrófila mista, em Curitiba, PR - Brasil. Revista Floresta, Curitiba, v. 1, n.32, p.3-16, 2002. 
ROSEIRA, D. S. Composição florística e estrutura fitossociológica do Bosque com Araucaria angustifolia (Bertol.) O. Ktze no Parque Estadual João Paulo II, Curitiba, Paraná. 1990. 110f. Dissertação ( Mestrado em Botânica) - Setor de Ciências Biológicas, Universidade Federal do Paraná, Curitiba, 1990.

SCHAAF, L. B.; FIGUEIREDO FILHO, A.; GALVÃO, F.; SANQUETTA, C. R.; LONGHI, S. J. Modificações florístico-estruturais de um remanescente de floresta ombrófila mista montana no período entre 1979 e 2000. Ciência Florestal, Santa Maria, v.16, n.3, p.271-291, 2006.

SEGER, C. D.; DLUGOSZ, F. L.; KURASZ, G.; MARTINEZ, D. T.; RONCONI, E.; MELO, L.A. N.; BITTENCOURT, S.M.; BRAND, M.A.; CARNIATTO, I. GALVÃO, F.; RODERJAN, C. V. Levantamento florístico e análise fitossociológica de um remanescente de floresta ombrófila mista localizado no município de Pinhais, Paraná-Brasil. Revista Floresta, Curitiba, v.35, n.2, p.291-302, mai-ago 2005.

SECRETARIA DE ESTADO DO MEIO AMBIENTE - SEMA. Resolução no 031 de 24 de agosto de 1998. Diário Oficial [do] Estado do Paraná. Curitiba, 25 ago. 1998.

SECRETARIA MUNICIAPAL DO MEIO AMBIENTE - SEMAFLOR. Prefeitura Municipal de Guarapuava. Disponível em: <htpp://www.prefeituramunicipalde guarapuava/>. Acesso em: 5 jan. 2010.

SILVA, C. da S.; MARCONI. L. P. Fitossociologia em uma floresta com araucária em Colombo - PR. Boletim de Pesquisa Florestal, Colombo, n.20, p.23-38, jun. 1990.

SILVA, D. W. Florística e Fitossociologia de dois remanescentes de Floresta Ombrófila Mista (Floresta com Araucária) e Análise de duas populações de Araucaria angustifolia (Bertol.) O. Kuntze na região de Guarapuava, PR. 2003. 160f. Tese (Doutorado em Ecologia) - Centro de Ciências Biológicas e da Saúde, Universidade Federal de São Carlos, São Carlos, 2003.

SILVA, D. W. A vegetação da Bacia do Rio das Pedras. In: BATTISTELLI, M. et al. (Org.). Proteção e manejo da Bacia do Rio das Pedras. Guarapuava: B \& D Ltda., 2004. p.91-99.

SONDA, C.; OLIVEIRA, E.A. de; LOPEZ, M. R. Q.; BONNET, B. Estudo fitossociológico de uma reserva florestal legal: conhecer para intervir. Cadernos da Biodiversidade, Curitiba, 60, v.2, n.1, p.62-72. jul. 1999.

SOUZA, V. C.; LORENZI, H. Botânica Sistemática - Guia ilustrado para identificação das famílias de Angiospermas da flora brasileira, baseado em APG II. 2. ed. Nova Odessa : Instituto Plantarum. 2008. 640 p.

VELOSO, H. P. Os grandes climaces do Brasil. I - Considerações sôbre os tipos vegetativos da região sul. Mem. Inst. Oswaldo Cruz, Rio de Janeiro, v.60, n.2, p.175-190, 1962. 
VELOSO, H. P.; RANGEL FILHO, A. L. P.; LIMA, J. C. A. Classificação da vegetação brasileira adaptada a um sistema universal. Rio de Janeiro: IBGE, 1991.

WATZLAWICK, L. F.; SANQUETTA, C. R.; VALÉRIO, A. F.; SILVESTRE, R. Caracterização da composição florística e estrutura de uma floresta ombrófila mista, no município de General Carneiro (PR). Ambiência, Guarapuava, v.1, n.2, p.229-237, 2005.

ZILLER, S. R.A estepe Gramíneo-Lenhosa no Segundo Planalto do Paraná:Diagnóstico Ambiental com Enfoque à Contaminação Biológica. 2000. 242f. Tese de Doutorado (Doutorado em Ciências Florestais) - Setor de Ciências Agrárias, Universidade Federal do Paraná, Curitiba, 2000. 